\section{PSQ-072 ADEQUACY OF ANTIDEPRESSANT MEDICATION IN ELDERLY PATIENTS}

'J Delgado Rodriguez*, 'L Perez Cordon, 'M Bitlloch Obiols, ${ }^{2} S$ Marin Rubio, 'L Campins Bernadas, 'V Aguilera Jimenez, 'T Gurrera Roig, ${ }^{3} \mathrm{M}$ Serra Prat. ${ }^{1}$ Hospital De Mataró, Pharmacy, Barcelona, Spain; ${ }^{2}$ Hospital Universitary Dexeus, Pharmacy, Barcelona, Spain; ${ }^{3}$ Hospital De Mataró, Research Unit, Barcelona, Spain

\subsection{6/ejhpharm-2020-eahpconf.389}

Background and importance Depression affects about 14-26\% of the elderly population. It is a frequent affective disorder and one of the main reasons for medical consultation. Elderly patients usually have several comorbidities that make polypharmacy a common issue. In the light of the above information, it is especially important that their antidepressant medication is adequate.

Aim and objectives To assess the adequacy of antidepressant treatment and the medication related factors associated with poor quality prescriptions in elderly patients diagnosed with depression.

Material and methods Elderly patients, defined as those aged $\geq 70$ years, diagnosed with depression in three primary care centres, from March 2018 to May 2018, were included. Demographic data (age and gender) and the treatment prescribed at the time of the study were collected from the electronic clinical history. Patients with antidepressant therapy and those without were analysed. Also, the adequacy of the prescriptions, consulting different psychogeriatric guides and taking into account the criteria repeated in two or more guides, and the reasons for poor quality prescriptions were studied.

Results The study included 170 patients, mean age 77.3 (7192) years and $81.2 \%$ were women. A total of $130(76.5 \%)$ patients were treated and 150 antidepressant prescriptions were analysed: $27(20.8 \%)$ patients were inappropriately treated and $27(18.0 \%)$ prescriptions were inadequate. Forty $(23.5 \%)$ patients were not treated and $16(40.0 \%)$ should have been treated with antidepressant therapy. In total, 43 $(25.3 \%)$ patients were not being adequately treated. The main reasons for the inadequate prescriptions were: $3(7.0 \%)$ overdosing, 1 (2.3\%) underdosing, $6(14.0 \%)$ incorrect duration, $17(39.5 \%)$ incorrect indication, including lack of treatment, and $16(37.2 \%)$ adverse effects.

Conclusion and relevance About 25\% of elderly patients with depression had inadequate prescriptions so it is important to choose an adequate treatment in order to reduce adverse effects and improve efficacy, especially in the treatment of a prevalent disease in a fragile population. Clinical pharmacists have an important role in the detection of inadequate medication in this group of patients.

\section{REFERENCES AND/OR ACKNOWLEDGEMENTS}

No conflict of interest.

\section{PSQ-073 USEFULNESS OF SALIVA IN THERAPEUTIC DRUG MONITORING OF CAFFEINE IN PRETERM INFANTS}

${ }^{1}$ AA Garcia Robles*, ${ }^{2} T$ Palanques Pastor, ${ }^{3} \mathrm{P}$ Sáenz González, ${ }^{3} \mathrm{~A}$ Solaz García, ${ }^{3}$ I Lara Cantón, ${ }^{3} \mathrm{C}$ Cháfer Pericás, ${ }^{2} \mathrm{JL}$ Poveda Andrés, ${ }^{3} \mathrm{M}$ Vento Torres. ${ }^{1}$ Hospital Universitari I Politècnic La Fe, Pharmacy-Neonatology, Valencia, Spain; ${ }^{2}$ Hospital Universitari I Politècnic La Fe, Pharmacy, Valencia, Spain; ${ }^{3}$ Hospital Universitari I Politècnic La Fe, Neonatology, Valencia, Spain

10.1136/ejhpharm-2020-eahpconf.390

Background and importance Apnoea of prematurity (AOP) is an alteration of the regulation of breathing and is very prevalent. The treatment of choice is caffeine but there is a lot of variability in its dosage, often using doses higher than recommended. Despite the wide safety range, pharmacokinetic monitoring may be necessary in certain cases.

Aim and objectives The goal was to establish the usefulness of saliva for monitoring serum caffeine levels non-invasively.

Material and methods This was a single centre, prospective, observational study. Premature patients admitted to the neonatology service between December 2017 and August 2018 being treated with caffeine for AOP were sequentially included on request for informed consent. Two paired samples of saliva-blood were collected per patient, at pre-dose time, taking advantage of routine blood withdrawals. Saliva was collected with cellulose sponges (Eyetec) in Eppendorf tubes and blood in dry tubes (Mini-collect). Salivary secretion stimulators were not used. The samples were centrifuged immediately after being collected and stored at $-20^{\circ} \mathrm{C}$. The determination was made by micro-extraction in solid phase in tube coupled to capillary liquid chromatography. We recorded date of birth, gestational age (GA), sex, weight, caffeine treatment regimen, renal function and concomitant drugs.

Results Forty-seven neonates were included, mean GA 27.8 (SD 2.36) weeks, birth weight $1.11 \pm 0.44 \mathrm{~kg}$ and $62 \%$ were male sex. Mean corrected GA on the day of determination was $31 \pm 2.7$ weeks and weight was $1.85 \pm 0.45 \mathrm{~kg}$. Administration was oral in 59\% and intravenous in 41\%. The mean concentration of caffeine in saliva was $16.35 \pm 9.21$ and in serum 19.28 $\pm 9.21 \mu \mathrm{g} / \mathrm{mL}$. Serum and saliva concentrations showed a strong correlation (Pearson's correlation $=0.83$, figure 1 ), which was higher with oral administration (0.90 vs 0.73 intravenous). Predictive model of linear regression of blood values was performed from saliva values. When comparing weight, GA, sex, caffeine dose $(\mathrm{mg} / \mathrm{kg})$ and concomitant drugs, no differences in correlation were observed through a multivariate analysis. No patient had kidney failure.

Conclusion and relevance Saliva determination is a reliable and non-invasive method for monitoring caffeine levels in preterm children with AOP. Correlation was higher when caffeine was administered orally, probably due to greater clinical stability when oral medication is administered.

\section{REFERENCES AND/OR ACKNOWLEDGEMENTS}

No conflict of interest.

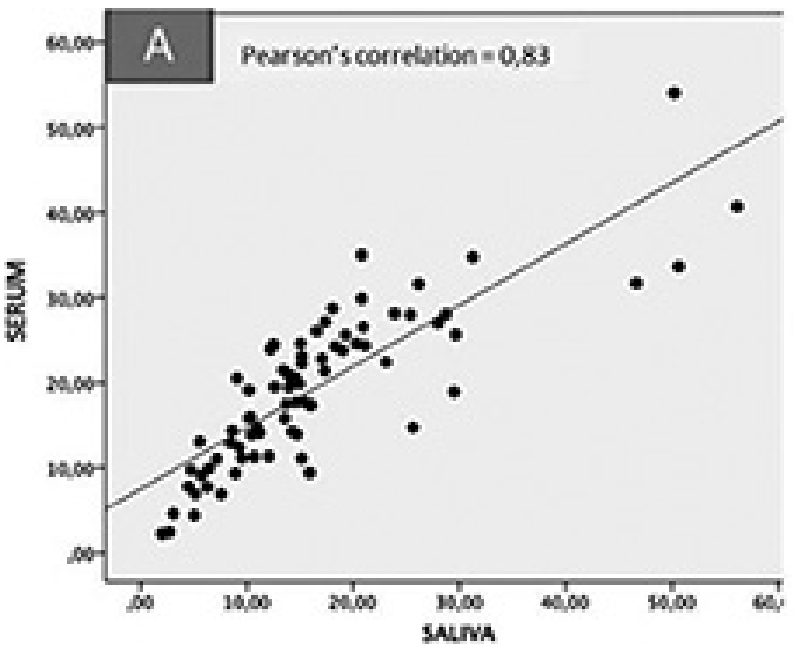

Abstract 5PSQ-073 Figure 1 Correlation between serum and saliva (intravenous and oral) levels 